\title{
Non-Stanley Bounds for Network Reliability*
}

\author{
JASON I. BROWN \\ Department of Mathematics, Statistics and Computing Science, Dalhousie University, Halifax, Canada \\ CHARLES J. COLBOURN \\ Department of Combinatorics and Optimization, University of Waterloo, Waterloo, Canada
}

Recelved May 17, 1993; Revised January 20, 1995

\begin{abstract}
Suppose that each edge of a connected graph $G$ of order $n$ is independently operational with probability $p$; the reliability of $G$ is the probability that the operational edges form a spanning connected subgraph. A useful expansion of the reliability is as $p^{n-1} \sum_{i=0}^{d} H_{i}(1-p)^{i}$, and the Ball-Provan method for bounding reliability relies on Stanley's combinatorial bounds for the $H$-vectors of shellable complexes. We prove some new bounds here for the $H$-vectors arising from graphs, and the results here shed light on the problem of characterizing the $H$-vectors of matroids.
\end{abstract}

Keywords: network reliability, H-vector, shellable complex, matroid, graph polynomial

\section{Introduction}

Let $G$ be a connected undirected graph, with possibly multiple edges and loops, of order $n$ and size $m$ (i.e. with $n$ vertices and $m$ edges). $\operatorname{Rel}(G, p)$ denotes the reliability of $G$, that is, the probability that $G$ is connected, where each edge of $G$ is independently operational with probability $p$. Much work has been done on calculating and approximating $\operatorname{Rel}(G, p)$ (whose determination is \#P-complete); see for example [9] for a survey. $\operatorname{Rel}(G, p)$ is always a polynomial in $p$ (the reliability polynomial of $G$ ) and can be expressed in a variety of ways (each with its own advantages to the estimation of reliability [9]):

$$
\begin{aligned}
\operatorname{Rel}(G, p) & =\sum_{i=n-1}^{m}(-1)^{i-n+1} S_{i} p^{i} \quad(S \text {-form }) \\
& =\sum_{i=0}^{m-n+1} F_{i} p^{m-i}(1-p)^{i} \quad(F \text {-form }) \\
& =\sum_{i=n-1}^{m} N_{i} p^{i}(1-p)^{m-i} \quad(N \text {-form }) \\
& =p^{n-1} \sum_{i=0}^{m-n+1} H_{i}(1-p)^{i} \quad(H \text {-form }) \\
& =1-\sum_{i=\lambda}^{m} C_{i} p^{m-i}(1-p)^{i} \quad(C \text {-form })
\end{aligned}
$$

${ }^{*}$ Research supported by NSERC Canada. 
( $\lambda$ denotes the edge connectivity of $G$ ). All the sequences $\left\langle S_{i}\right\rangle,\left\langle F_{i}\right\rangle,\left\langle N_{i}\right\rangle,\left\langle H_{i}\right\rangle$ and $\left\langle C_{i}\right\rangle$ consist of nonnegative integers (c.f. [9]). These sequences have combinatorial significance. $N_{t}$ is the number of subsets of edges of size $i$ that form a spanning connected subgraph of $G$. $C_{i}$ is the number of cut sets of size $i . F_{i}$ is the number of subsets of edges of size $i$ whose removal do not disconnect $G$.

In fact, some results on the combinatorial properties of simplicial complexes play a role in reliability, so we introduce some salient notation. A complex $\mathcal{C}$ on a 'ground' set $S$ is a non-empty collection of subsets of $S$ that is closed under containment. The subsets of $S$ that belong to $\mathcal{C}$ are called faces or simplices, and the maximal faces are called facets or bases. The face vector (or simply the $F$-vector) of $\mathcal{C},\left\langle F_{i}\right\rangle$, is the sequence in which $F_{i}$ is the number of faces of cardinality $i$ in $\mathcal{C}$. A complex $\mathcal{C}$ is pure if all facets are of the same cardinality. The dimension of a pure complex is the cardinality of any facet (this definition is one greater than the dimension often defined for pure complexes, but we find this definition simpler here). For a pure complex $\mathcal{C}$ of dimension $d$ with $F$-vector $\left(F_{0}, \ldots, F_{d}\right)$, the $H$-vector [2, $14],\left(H_{0}, \ldots, H_{r}\right)$ has terms given by

$$
H_{i}=\sum_{j=0}^{i}(-1)^{i-j}\left(\begin{array}{l}
d-j \\
d-i
\end{array}\right) F_{j} \quad(0 \leq i \leq d) .
$$

$H$-vectors have received considerable attention in polytope theory, in particular in connection with the Upper Bound Theorem.

The $H$-vector can also be interpreted in the following sense. For any sets $L$ and $U$ with $L \subseteq U$, the interval $[L, U]$ is $\{S: L \subseteq S \subseteq U\}$. A pure complex $\mathcal{C}$ is shellable if its facets can be ordered as $\sigma_{1}, \ldots, \sigma_{m}$ where for any $k \in\{2, \ldots, m\}$,

$$
\bar{\sigma}_{k} \cap\left(\bigcup_{i=1}^{k-1} \bar{\sigma}_{i}\right)
$$

is a purely $(d-1)$-dimensional complex ( $\bar{\sigma}$ denotes the set of all subsets of $\sigma$ ); a pure complex $\mathcal{C}$ is partitionable if its simplices can be partitioned into intervals

$$
\left[L_{1}, U_{1}\right], \ldots,\left[L_{\ell}, U_{\ell}\right]
$$

where each $U_{i}$ is a facet of $\mathcal{C}$. It is well known (c.f. $\left.[9,3]\right)$ that any shellable complex is partitionable, and in the case of a partitionable complex,

$$
H_{l}=\left|\left\{j:\left|L_{j}\right|=i\right\}\right| .
$$

Tutte's seminal paper [15] on the dichromatic polynomial employed partitions of graphic matroids using internal and external activities of edges; when extended to matroids, Tutte's observations establish that every matroid is partitionable (see also [9, 3]). The $H$-vector of any matroid has no internal zeros, and the last nonzero term is $H_{r}$, where $r$ is the dimension of the matroid minus the number of coloops (see $[3,7]$ ); this last term is the absolute value of the Euler characteristic of the matroid [3].

Stanley [13] completely characterizes the $H$-vectors of shellable complexes. Let $\left(H_{0}, \ldots\right.$, $\left.H_{d}\right)$ be the $H$-vector of such a complex. Fix $k \in\{0, \ldots, d-1\}$. The $k$-canonical form of a positive integer $r$ is the integer-valued vector $\left(a_{k}, a_{k-1}, \ldots, a_{j}\right)$, where

$$
r=\left(\begin{array}{l}
a_{k} \\
k
\end{array}\right)+\left(\begin{array}{l}
a_{k-1} \\
k-1
\end{array}\right)+\cdots+\left(\begin{array}{c}
a_{j} \\
j
\end{array}\right)
$$


and

$$
a_{k}>a_{k-1}>\cdots a_{j} \geq j \geq 1
$$

(such a representation exists for all $r$ and $k$ and is unique). $r^{\langle t / k\rangle}$, the $(i, k)$ th $u p p e r$ pseudopower of $r$, is then defined to be

$$
r^{\langle l / k\rangle}=\left(\begin{array}{c}
a_{k}-k+i \\
i
\end{array}\right)+\left(\begin{array}{c}
a_{k-1}-k+i \\
i-1
\end{array}\right)+\cdots+\left(\begin{array}{c}
a_{j}-k+i \\
i-k+j
\end{array}\right)
$$

it is not hard to see that $r^{\langle t / k\rangle}$ is a nondecreasing function of $r, r^{\langle t+2 / i\rangle}=$ $\left(r^{(i+1 / i\rangle}\right)^{\langle i+2 / i+1\rangle}$, and $r=\left(r^{\langle i / j\rangle}\right)^{\langle j / i\rangle}$. Then a necessary [12] and sufficient [13] condition for a nonnegative integer vector $\left\langle H_{0}, \ldots, H_{d}\right\rangle$ to be an $H$-vector of some shellable complex is that

$$
H_{0}=1
$$

and

$$
H_{i+1} \leq H_{i}^{\langle i+1 / i\rangle}
$$

for $i=1, \ldots, d-1$. We call such bounds Stanley bounds.

Stanley bounds do not put any lower bounds for the tail of an $H$-vector in terms of the initial sequence.

One of the best techniques for estimating reliability is the Ball-Provan method [1] (see also [9]), which relies on Stanley's bounds for the terms in the $H$-vectors of shellable complexes (as applied to cographic matroids). For a connected graph $G$, let $\mathcal{F}(G)$ be the complex on the ground set $E(G)$ of edges of $G$ whose simplices are the subsets of $E(G)$ whose removal does not disconnect $G ; \mathcal{F}(G)$ is in fact the cographic matroid of $G$, and if $G$ has $n$ vertices and $m$ edges, then $\mathcal{F}(G)$ has dimension $m-n+1$. The sequences $\left\langle F_{l}\right\rangle$ and $\left\langle H_{i}\right\rangle$ from the $F$ and $H$ forms of the reliability polynomial of $G$ are in fact the $F$-vector and $H$-vector of the pure complex $\mathcal{F}(G)$, and we simply call these the $F$ - and $H$-vectors of the graph $G$. We thus see at once that $\left\langle H_{i}\right\rangle$ is a sequence of nonnegative integers. It is this connection that allowed Ball and Provan to apply Stanley's bounds to estimate reliability efficiently.

One can improve upon the Stanley bounds, as applied to network reliability, in a number of ways. First, one can employ algorithms that calculate bounds on coefficients given a specific input graph. Second, one can employ transformations that have a predictable effect on the $H$-vector; when such transformations reduce the graph to a simply analyzed structure (such as a series-parallel graph), one can extract bounds on the original $H$-vector from that of the reduced graph. This approach is taken, for example, in [8] and [4]. The third method is to develop inequalities among the terms of the $H$-vector that hold for any cographic matroid. It is the third of these approaches that we examine in this paper. We develop here new bounds for $H$-vectors of cographic matroids, and apply the results to bounding reliability. Björner [3] states that little is known about characterizing the $H$-vector of matroids. Some of the results in this paper shed light on this problem as they extend also to new general inequalities on the $H$-vectors of matroids.

We use the following notation. A graph $G=(V(G), E(G))$ may have loops or multiple edges; a simple graph has no loops or multiple edges. The order of $G$ is its number of vertices, and the size of $G$ is its number of edges. Given an edge $e$ of $G$ that is not a 
loop, $G-e$ denotes the graph formed from $G$ by deleting edge $e$, and $G \cdot e$ denotes the graph formed from $G$ by contracting edge $e$ (and deleting $e$ ). An edge $e$ of a connected graph $G$ is a bridge if $G-e$ is disconnected. $G$ is a block if it is 2-connected, that is $G$ is connected and the removal of any vertex also leaves a connected graph. The girth of a graph $G, \gamma(G)$, is the length of its smallest cycle (if $G$ is acyclic, we set $\gamma(G)=\infty$ ), and the circumference of $G$ is the length of the longest cycle. If $G$ and $H$ are graphs then we write $G \equiv H$ if by contracting bridges and deleting loops the two graphs can be made isomorphic. The $K_{1}$-bond of graphs $G$ and $H$ is formed from vertex disjoint copies of $G$ and $H$ by identifying a vertex of each; we write $G \circ H$ for any such graph. A cycle of length $n \geq 2$ is denoted by $C_{n}$ ( $C_{2}$ is a set of two parallel edges). A $\theta$-graph consists of two vertices of degree 3 joined by three internally disjoint paths. A multiple of a graph $G$ is a graph $G^{\prime}$ on the same vertex set formed by replacing each edge of $G$ by a nonempty set of parallel edges. For $k \geq 1, k G$ is the multiple of $G$ formed by replacing each edge of $G$ by $k$ parallel edges. We refer the reader to [16] for standard terminology on matroids. Finally, we denote the last nonzero term in the $H$-vector of a graph $G$ by $H_{d}(d=d(G)$ is the dimension of the associated cographic matroid if the graph is loopless, and if $G$ is not loopless, $d$ is the dimension of its cographic matroid minus the number of loops in $G$ ); in either case, $G$ is said to have dimension $d$. (Loops have no effect on reliability and so are easily treated, either by defining dimension as above or by permitting the $H$-vector to have trailing zeros. We adopt the first approach here to simplify the statement of later results.)

It is clear from $\operatorname{Rel}(G, p)=(1-p) \operatorname{Rel}(G-e, p)+p \operatorname{Rel}(G \cdot e, p)$ that for all $i=1, \ldots, d(G)$,

$$
H_{i}(G)=H_{i-1}(G-e)+H_{i}(G \cdot e) .
$$

In the next section we derive new bounds for weighted sums of terms of the $H$-vectors of both graphs and matroids, primarily through a combinatorial interpretation of the $H_{i}$ 's as counting spanning trees (or bases of a matroid) with a certain property. The following section produces inequalities among the $H_{i}$ 's for graphs, with a precise determination in each case of when equality holds. In the two final sections, we focus on the last non-zero term of the $H$-vector, which has been called the domination number of a graph, and provide non-Stanley bounds there as well.

\section{Non-Stanley $\boldsymbol{H}$-bounds from external activity}

An explicit partition of a cographic matroid (see $[3,9]$ ) is as follows. Let $\prec$ be a fixed linear order of the ground set $E$ of a matroid $M$. In particular, for the cographic matroid of a connected graph $G$ let $\prec$ be a fixed linear order of $E(G)$, let $\mathcal{T}$ denote the set of all spanning trees of $G$, and for an edge $e$ of $G$, call $e$ externally active with respect to $T$ if $e \notin T$ but $e$ is the least edge of the unique cycle in $T+e$. $\operatorname{NEA}(T)$ denotes the edges of $G$ not in $T$ that are not externally active with respect to $T$. Then

$$
\{[\operatorname{NEA}(T), E-T]: T \in \mathcal{T}\}
$$

is a partition of the cographic matroid of $G$. Thus $H_{i}=|\{T \in \mathcal{T}:|\operatorname{NEA}(T)|=i\}|$. 
In [11] the following inequality for $H$-vectors of matroids was shown by algebraic methods:

$$
\sum_{i \leq k} H_{i} \leq \sum_{i \geq d-k} H_{i} .
$$

As a corollary, this inequality holds for $H$-vectors of graphs as well. It is easy to see that equality holds for graphs $G$ for all $k$ if $G$ is a multiple of a tree, since the $H$-vector of a set of parallel edges is symmetric, and hence so (by products) is the multiple of any tree. We prove that equality only holds in these cases, and extend Hibi's result with a purely combinatorial argument that considers external activity.

Theorem 1 Let $G$ be a loopless graph of dimensiond with a cycle of length $\ell \geq 2$, and let the $H$-vector of $G$ be $\left(H_{0}, \ldots, H_{d}\right)$. Then for any $k \leq d-1$,

$$
\sum_{i \leq k} H_{i}+(\ell-2) \leq \sum_{i \geq d-k} H_{i}
$$

Proof: Let $e_{1} \prec e_{2} \prec \cdots \prec e_{m}$ be a fixed linear order of $E-E(G)$. We let $\mathcal{T}_{\prec, i, j}(G)$ denote the set of spanning trees $T$ of $G$ such that there are exactly $i$ externally active edges in $(G, \prec)$ and exactly $j$ externally active edges in $(G, \succ)$, the reverse ordering; in other words, there are exactly $i$ edges $e$ of $G$ not in $T$ for which $e$ is the least edge in the unique cycle of $T+e$, and there are exactly $j$ edges $e$ of $G$ not in $T$ for which $e$ is the greatest edge in the unique cycle of $T+e$. Since $0 \leq i, j \leq d$ and $G$ is loopless, $i+j \leq d$. Also note that as $H_{i}$ is the number of spanning trees $T$ such that exactly $i$ edges of $G-T$ are not externally active with respect to $T$ (and hence exactly $d-i$ edges of $G-T$ are externally active with respect to $T$ ),

$$
\begin{aligned}
H_{t} & =\sum_{J}\left|\mathcal{T}_{\prec, d-t, j}(G)\right| \\
& =\sum_{J}\left|\mathcal{T}_{\succ, d-t, j}(G)\right| \\
& =\sum_{J}\left|\mathcal{T}_{\prec, j, d-i}(G)\right|
\end{aligned}
$$

since the $H$-vector is independent of the ordering of the graph. Thus

$$
\begin{aligned}
\sum_{i=d-k}^{d} H_{i} & =\sum_{i=0}^{k} \sum_{j}\left|\mathcal{T}_{<, i, j}(G)\right| \\
& =\sum_{i=0}^{k} \sum_{j}\left|\mathcal{T}_{<, j, i}(G)\right| \\
& =\sum_{j=d-k}^{d} \sum_{i=0}^{k}\left|\mathcal{T}_{<, j, i}(G)\right|+\sum_{j=0}^{d-k-1} \sum_{i=0}^{k}\left|\mathcal{T}_{<, j, i}(G)\right| \\
& =\sum_{i=0}^{k} H_{i}+\sum_{j=0}^{d-k-1} \sum_{i=0}^{k}\left|\mathcal{T}_{<, j, i}(G)\right|
\end{aligned}
$$


Now for any unicyclic subgraph (i.e. a subgraph with exactly one cycle) $G^{\prime}$ of $G$ with a cycle of length $\ell \geq 2$,

$$
\left|\mathcal{T}_{\prec, 0,0}\left(G^{\prime}\right)\right|=\ell-2
$$

for any ordering $\prec$ on the graph. Let $0 \leq k \leq d-1$. Let the edges not in $G^{\prime}$ be $f_{1}, \ldots, f_{d-1}$. Order the edges of $G$ such that $f_{1}, \ldots, f_{k}$ are smaller than those of $G^{\prime}$, which are in turn smaller than $f_{k+1}, \ldots, f_{d-1}$. It is easy to verify that if $T \in \mathcal{T}_{<, i, j}\left(G^{\prime \prime}\right)$ is a spanning tree of a spanning subgraph $G^{\prime \prime}$ of $G$ and $e$ is an edge of $G$ not in $T$, then by setting $e$ smaller than all edges of $G^{\prime \prime}, T \in \tau_{<, i+1, j}\left(G^{\prime \prime}+e\right)$; and conversely if we set $e$ larger than all edges of $G^{\prime \prime}$. Thus we see inductively that

$$
\left|\mathcal{T}_{<, d-k-1, k}(G)\right| \geq\left|\mathcal{T}_{<, 0,0}\left(G^{\prime}\right)\right|=\ell-2
$$

and substituting this into (1), we derive

$$
\sum_{i \leq k} H_{i}+(\ell-2) \leq \sum_{i \geq d-k} H_{i} .
$$

The results can be improved. For example, if $G$ has a $\theta$-subgraph $G^{\prime}$ with edge lengths $\ell_{1}, \ell_{2}$, and $\ell_{3}$, then

$$
\left|\mathcal{T}_{<, 0,0}\left(G^{\prime}\right)\right|=\sum_{i<j}\left(\ell_{i}-1\right)\left(\ell_{j}-1\right)
$$

and

$$
\left|\mathcal{T}_{<, 0,1}\left(G^{\prime}\right)\right|=\left|\mathcal{T}_{<, 1,0}\left(G^{\prime}\right)\right|=\left(\sum_{i} \ell_{i}\right)-3
$$

and if $G$ has a subgraph $G^{\prime}$ consisting of two vertex-disjoint cycles of lengths $n_{1}$ and $n_{2}$, then

$$
\left|\mathcal{T}_{<, 0,0}\left(G^{\prime}\right)\right|=\left(n_{1}-2\right)\left(n_{2}-2\right)
$$

and

$$
\left|\mathcal{T}_{\prec, 0,1}\left(G^{\prime}\right)\right|=\left|\mathcal{T}_{<, 0,1}\left(G^{\prime}\right)\right|=n_{1}+n_{2}-4,
$$

we can then pull these up through the ranks to improve the gap between $\sum_{i=0}^{k} H_{i}$ and $\sum_{i=d-k}^{d} H_{i}$.

The previous theorem extends to matroids in the following way. An element $e$ of a matroid $M$ is externally active with respect to basis $B$ of $M$ if $e \in E-B$ and $e$ is the smallest element of the unique circuit in $B+e \equiv B \cup\{e\}$. We let $\mathrm{NEA}_{M, \prec}(B)$ denote the set of edges of $E-B$ that are not externally active with respect to $B$. Then

$$
\left\{\left[\operatorname{NEA}_{M, \prec}(B), E-B\right]: B \text { is a basis of } M\right\}
$$

forms a partition of $M^{*}$, the dual matroid of $M$ (see, for example, [2] or [10]). A similar argument to the proof of Theorem 1 proves an strengthening of Hibi's results on matroids: 
Theorem 2 Let $M$ be a matroid of dimension d without coloops, and suppose that $M$ has a cocircuit of length $\ell \geq 2$. Let the $H$-vector of $M$ be $\left(H_{0}, \ldots, H_{d}\right)$. Then for any $k \leq d-1$,

$$
\sum_{i \leq k} H_{i}+(\ell-2) \leq \sum_{i \geq d-k} H_{l}
$$

We return to graphs. It is easy to see that $\sum H_{i}$ is simply the number of spanning trees of $G$ (and hence is computable in polynomial time from the well known Matrix Tree Theorem). By considering all possible linear orderings of the edges we can obtain a new equation involving $\sum i H_{l}$.

Theorem 3 For any connected loopless graph $G$ of dimension d,

$$
\sum_{i=0}^{d} i H_{i}=\sum_{T \in \mathcal{T}} \sum_{e \in E(G)-T} \frac{c(T, e)-1}{c(T, e)}
$$

where $\mathcal{T}$ is the set of all spanning trees of $G$, and for a spanning tree $T$ of $G$ and an edge $e$ of $G$ not in $T, c(T, e)$ is the length of the unique cycle in $T+e$.

Proof: For each spanning tree $T$ of $G$, each edge $e$ of $G$ not in $T$ and each linear order $\sigma$ of $E(G)$, let $f(T, e, \sigma)$ be 1 if $e$ is not externally active with respect to $T$ under the ordering $\sigma$, and 0 otherwise. Now for a fixed tree $T$ and edge $e \in E(G)-T$, for how many orderings $\sigma$ is $e$ externally active with respect to $T$ ? There are

$$
\left(\begin{array}{c}
m \\
c(T, e)
\end{array}\right)
$$

positions for the edges of the unique cycle of $T+e$ with respect to $\sigma$; there are

$$
(m-c(T, e)) !
$$

ways to arrange the remaining edges, and there are

$$
(c(T, e)-1) !
$$

ways to arrange the edges of the cycle so that $e$ is externally active. Thus we have

$$
\begin{aligned}
\sum_{T \in \mathcal{T}} \sum_{e \in E(G)-T} \sum_{\sigma} f(T, e, \sigma)= & \sum_{T \in \mathcal{T}} \sum_{e \in E(G)-T}\left(\begin{array}{c}
m \\
c(T, e)
\end{array}\right) \cdot(m-c(T, e)) ! \\
& \cdot(c(T, e)-1) \cdot(c(T, e)-1) ! \\
= & \sum_{t \in \mathcal{T}} \sum_{e \in E(G)-T} \frac{m ! \cdot(c(T, e)-1)}{c(T, e)} .
\end{aligned}
$$

On the other hand, by reversing the order of summation, we get

$$
\sum_{\sigma} \sum_{T \in \mathcal{T}} \sum_{e \in E(G)-T} f(T, e, \sigma)=m ! \sum_{i=0}^{d} i H_{l},
$$


so we have

$$
\sum_{i=0}^{d} i H_{i}=\sum_{T \in T} \sum_{e \in E(G)-T} \frac{c(T, e)-1}{c(T, e)}
$$

Corollary 4 If a connected loopless graph $G$ of dimension $d$ has order $n, t$ spanning trees, girth at least $g$ and circumference at most $c$ then

$$
\frac{g-1}{g} t d \leq \sum_{i=0}^{d} i H_{i} \leq \frac{c-1}{c} t d .
$$

In particular, if $G$ is simple then

$$
\frac{2}{3} t d \leq \sum_{i=0}^{d} i H_{i} \leq\left(1-\frac{1}{n}\right) t d
$$

Again, the arguments yield a result on the $H$-vectors of matroids.

Corollary 5 If a matroid $M$ with no coloops has order $n$ (i.e. its base set has $n$ elements), $t$ bases and smallest and largest lengths of cocircuits $g$ and $\ell$ respectively, then

$$
\frac{g-1}{g} t d \leq \sum_{i=0}^{d} i H_{i} \leq \frac{\ell-1}{\ell} t d \text {. }
$$

By a similar argument, we prove the following:

Theorem 6 If a connected loopless graph $G$ of dimension d has order $n, t$ spanning trees, girth at least $g$ and circumference at most $c$ then

$$
\sum_{i=0}^{d}\left(\begin{array}{l}
i \\
2
\end{array}\right) H_{i} \geq t \cdot\left(\begin{array}{l}
d \\
2
\end{array}\right) \cdot \min \left\{\left(1-\frac{1}{g}\right)^{2}, 1-\frac{2}{g}+\frac{3}{c^{2}}\right\}
$$

and

$$
\sum_{i=0}^{d}\left(\begin{array}{l}
i \\
2
\end{array}\right) H_{i} \leq t \cdot\left(\begin{array}{l}
d \\
2
\end{array}\right) \cdot \max \left\{\left(1-\frac{1}{c}\right)^{2}, 1-\frac{2}{c}+\frac{3}{g^{2}}\right\}
$$

Proof: For a given linear order $\sigma$ of $E(G)$ and spanning tree $T$ of $G$, let $\operatorname{EA}_{\sigma}(T)$ denote the set of externally active of $G$ with respect to $T$ and the ordering $\sigma$, and let $\operatorname{NEA}_{\sigma}(T)=E(G)-T-\operatorname{EA}_{\sigma}(T)$. Let $f\left(e_{1}, e_{2}, T, \sigma\right)=1$ if $e_{1}, e_{2} \in \operatorname{NEA}_{\sigma}(T)$, and let $f\left(e_{1}, e_{2}, T, \sigma\right)=0$ otherwise. Consider the sum

$$
\sum_{T \in T} \sum_{e_{1}, e_{2} \in E(G)-T} \sum_{\sigma} f\left(e_{1}, e_{2}, T, \sigma\right) .
$$

For a given spanning tree $T \in \mathcal{T}$ and edges $e_{1}, e_{2} \in E(G)-T$, for how many $\sigma$ 's are $e_{1}, e_{2} \notin \operatorname{NEA}_{\sigma}(T)$ ? There are two cases for $T+e_{1}+e_{2}$. 
Case 1 ( $T+e_{1}+e_{2}$ contains two edge disjoint cycles). Let the two cycles be $C_{1}$ and $C_{2}$ with lengths $\ell_{1}$ and $\ell_{2}$ respectively. Note that $g \leq \ell_{1}, \ell_{2} \leq c$. Now the number of orders of $E\left(C_{1}\right) \cup E\left(C_{2}\right)$ such that $e_{1}, e_{2}$ are not externally active is

$$
\begin{aligned}
g\left(T, e_{1}, e_{2}\right) & =\left(\ell_{1}-1\right) \cdot\left(\ell_{1}-1\right) ! \cdot\left(\ell_{2}-1\right) \cdot\left(\ell_{2}-1\right) ! \cdot\left(\begin{array}{c}
\ell_{1}+\ell_{2} \\
\ell_{1}
\end{array}\right) \\
& =m\left(T, e_{1}, e_{2}\right) ! \cdot\left(1-\frac{1}{\ell_{1}}\right)\left(1-\frac{1}{\ell_{2}}\right)
\end{aligned}
$$

where $m\left(T, e_{1}, e_{2}\right)=\ell_{1}+\ell_{2}$ is the number of edges in $C_{1} \cup C_{2}$.

Case $2\left(T+e_{1}+e_{2}\right.$ contains a $\theta$-graph). Let the $\theta$-subgraph of $T+e_{1}+e_{2}$ be denoted by $\Theta$ and have vertex disjoint paths $P_{1}, P_{2}, P_{3}$ of lengths $\ell_{1}, \ell_{2}, \ell_{3}$ respectively. For $i \neq j$, $g \leq \ell_{i}+\ell_{j} \leq c$. Without loss we can assume that $e_{1} \in P_{1}$ and $e_{2} \in P_{2}$. Now the number of orders of $E(\Theta)$ such that $e_{1}, e_{2}$ are not externally active is

$$
\begin{aligned}
g\left(T, e_{1}, e_{2}\right) \equiv & \left(\ell_{1}+\ell_{2}+\ell_{3}\right) !-\left(\ell_{1}+\ell_{3}-1\right) ! \ell_{2} !\left(\begin{array}{c}
\ell_{1}+\ell_{2}+\ell_{3} \\
\ell_{2}
\end{array}\right) \\
& -\left(\ell_{2}+\ell_{3}-1\right) ! \ell_{1} !\left(\begin{array}{c}
\ell_{1}+\ell_{2}+\ell_{3} \\
\ell_{1}
\end{array}\right) \\
& +\left(\ell_{2}+\ell_{3}-1\right) !\left(\ell_{1}-1\right) !\left(\begin{array}{c}
\ell_{1}-1+\ell_{2}+\ell_{3} \\
\ell_{1}-1
\end{array}\right) \\
& +\left(\ell_{1}+\ell_{3}-1\right) !\left(\ell_{2}-1\right) !\left(\begin{array}{c}
\ell_{2}-1+\ell_{1}+\ell_{3} \\
\ell_{2}-1
\end{array}\right) \\
= & m\left(T, e_{1}, e_{2}\right) !\left(1-\frac{1}{\ell_{1}+\ell_{3}}-\frac{1}{\ell_{2}+\ell_{3}}\right. \\
& \left.\quad+\frac{1}{m\left(T, e_{1}, e_{2}\right)}\left(\frac{1}{\ell_{2}+\ell_{3}}+\frac{1}{\ell_{1}+\ell_{3}}\right)\right) .
\end{aligned}
$$

where $m\left(T, e_{1}, e_{2}\right)=\ell_{1}+\ell_{2}+\ell_{3}$ is the number of edges in $\Theta$.

Now we have

$$
\begin{aligned}
& \sum_{T \in \mathcal{T}} \sum_{e_{1}, e_{2} \in E(G)-T} \sum_{\sigma} f\left(e_{1}, e_{2}, T, \sigma\right) \\
& =\sum_{T \in \mathcal{T}} \sum_{e_{1}, e_{2} \in E(G)-T} g\left(T, e_{1}, e_{2}\right) \cdot\left(m-m\left(T, e_{1}, e_{2}\right)\right) ! \cdot\left(\begin{array}{c}
m \\
m\left(T, e_{1}, e_{2}\right)
\end{array}\right) \\
& =m ! \sum_{T \in \mathcal{T}} \sum_{e_{1}, e_{2} \in E(G)-T} \frac{g\left(T, e_{1}, e_{2}\right)}{\left(m\left(T, e_{1}, e_{2}\right)\right) !} .
\end{aligned}
$$

In Case 1,

$$
\left(1-\frac{1}{g}\right)^{2} \leq \frac{g\left(T, e_{1}, e_{2}\right)}{\left(m\left(T, e_{1}, e_{2}\right)\right) !} \leq\left(1-\frac{1}{c}\right)^{2}
$$

and in Case 2,

$$
1-\frac{2}{g}+\frac{3}{c^{2}} \leq \frac{g\left(T, e_{1}, e_{2}\right)}{\left(m\left(T, e_{1}, e_{2}\right)\right) !} \leq 1-\frac{2}{c}+\frac{3}{g^{2}}
$$


Thus

$$
\begin{aligned}
& \sum_{T \in \mathcal{T}} \sum_{e_{1}, e_{2} \in E(G)-T} \sum_{\sigma} f\left(e_{1}, e_{2}, T, \sigma\right) \\
& \geq\left(\begin{array}{l}
d \\
2
\end{array}\right) \cdot t \cdot m ! \cdot \min \left\{\left(1-\frac{1}{g}\right)^{2}, 1-\frac{2}{g}+\frac{3}{c^{2}}\right\}
\end{aligned}
$$

and

$$
\begin{aligned}
& \sum_{T \in \mathcal{T}} \sum_{e_{1}, e_{2} \in E(G)-T} \sum_{\sigma} f\left(e_{1}, e_{2}, T, \sigma\right) \\
& \quad \leq\left(\begin{array}{l}
d \\
2
\end{array}\right) \cdot t \cdot m ! \cdot \max \left\{\left(1-\frac{1}{c}\right)^{2}, 1-\frac{2}{c}+\frac{3}{g^{2}}\right\} .
\end{aligned}
$$

By reversing the sums,

$$
\sum_{T \in \mathcal{T}} \sum_{e_{1}, e_{2} \in E(G)-T} \sum_{\sigma} f\left(e_{1}, e_{2}, T, \sigma\right)=m ! \sum_{i=0}^{d}\left(\begin{array}{l}
i \\
2
\end{array}\right) H_{i}
$$

so we have

$$
\sum_{i=0}^{d}\left(\begin{array}{l}
i \\
2
\end{array}\right) H_{i} \geq t \cdot\left(\begin{array}{l}
d \\
2
\end{array}\right) \cdot \min \left\{\left(1-\frac{1}{g}\right)^{2}, 1-\frac{2}{g}+\frac{3}{c^{2}}\right\}
$$

and

$$
\sum_{i=0}^{d}\left(\begin{array}{l}
i \\
2
\end{array}\right) H_{i} \leq t \cdot\left(\begin{array}{l}
d \\
2
\end{array}\right) \cdot \max \left\{\left(1-\frac{1}{c}\right)^{2}, 1-\frac{2}{c}+\frac{3}{g^{2}}\right\}
$$

Considering instead externally active pairs of edges, we can also show the following result (the details are omitted).

Theorem 7 If a connected loopless graph $G$ of dimensiond has order $n, t$ spanning trees, girth at least $g$ and circumference at most $c$ then

$$
\sum_{i=0}^{d}\left(\begin{array}{c}
d-i \\
2
\end{array}\right) H_{i} \geq t \cdot\left(\begin{array}{l}
d \\
2
\end{array}\right) \cdot \frac{1}{c^{2}}
$$

and

$$
\sum_{i=0}^{d}\left(\begin{array}{c}
d-i \\
2
\end{array}\right) H_{i} \leq t \cdot\left(\begin{array}{l}
d \\
2
\end{array}\right) \cdot \frac{3}{g^{2}}
$$

We point out one other inequality (though not related to external activity) of $H$-vectors of cographic matroids, which was proved in [7] in conjunction with an investigation of the roots of reliability polynomials. 
Theorem 8 For any $H$-vector $\left(H_{0}, \ldots, H_{d}\right)$ of a connected loopless graph, any real number $b \geq 1$, and any integer $j \in\{0, \ldots, d\}$,

$$
\sum_{i=0}^{j}(-b)^{i} H_{i} \begin{cases}\geq 0 & \text { if } j \text { is even } \\ \leq 0 & \text { if } j \text { is odd }\end{cases}
$$

These inequalities are strict if $b>1$.

We derive the following result.

Corollary 9 If $\left(H_{0}, \ldots, H_{d}\right)$ is the $H$-vector of a connected loopless graph with exactly $t$ spanning trees, then for all $i<d$,

$$
\sum_{j=0}^{\lfloor/ 2\rfloor} H_{i}^{<(i-2 j) / i>} \leq \frac{t}{2} .
$$

Proof: Consider the sum

$$
\sum_{j=0}^{i+1}(-1)^{j} H_{j}
$$

By the previous theorem, this sum has sign $(-1)^{i+1}$ or 0 , and hence

$$
\sum_{j=0}^{\lfloor l / 2\rfloor} H_{l-2 j} \leq \sum_{j=0}^{\lfloor(i+1) / 2\rfloor} H_{l+1-2 j}
$$

and since $\sum_{j=0}^{d} H_{j}=t$, we have

$$
\sum_{j=0}^{\lfloor i / 2\rfloor} H_{i-2 \jmath} \leq t / 2 .
$$

The Stanley bound and the elementary properties of pseudopowers mentioned in the first section ensure that $H_{i-2 \jmath} \geq H_{i}^{\langle(i-2 j) / i\rangle}$, to complete the proof.

This corollary is of use in bounding individual terms in the $H$-vector of a graph knowing only the number of spanning trees. One striking example is obtained by examining the $H$-vector of the 1979 Arpanet (see [8]). That network has 272,816,563,831 trees, and the best lower and upper bounds found by network transformations for $H_{11}$ are 13,884,089,682 and $185,684,104,966$ respectively. Corollary 9 , on the other hand, ensures that $H_{11}$ cannot exceed $103,425,922,079$; this eliminates $47.8 \%$ of the gap between the old lower and upper bounds. Naturally, knowing lower bounds on coefficients $H_{i}$ for $i \geq 12$ in this case can be used to obtain a tighter inequality yet. 


\section{Other general non-Stanley bounds}

Recall that the $H$-vector of any graph has no internal zeros, and the last nonzero term is $H_{d}$, where $d$ is the dimension of the graph. It is clear that $H_{1} \geq 1=H_{0}$ when the dimension is at least 1 , and in fact if $G$ has order $n$ and $\ell$ bridges, then $H_{1}=n-1-\ell$. We now characterize when $H_{2} \geq H_{1}$, and in particular when equality holds.

Theorem 10 If $G$ is a graph of dimension at least 2 then

$$
H_{2}(G) \geq H_{1}(G)
$$

unless $G \equiv C_{p} \circ C_{q}$ where $p=2$. Equality holds iff $G$ is a one of

- $k K_{2}(k \geq 2)$,

- $k K_{2} \circ C_{l}(k, l \geq 3)$

- $C_{l}(l \geq 3)$ with other edges parallel to one of the edges of $C_{l}$,

- $C_{2} \circ C_{2} \circ C_{2}$, or

- $C_{3} \circ C_{3}$

(see Fig. 1).

Proof: Without loss, $G$ is loopless and 2-edge connected, as the contraction of any bridge leaves the $H$-vector unchanged, but reduces the number of bridges. We proceed by induction on $n=|V(G)|$. Assume that $G \not \equiv C_{p} \circ C_{q}$ where $p=2$.

If $n=1$, the result is trivial as $\operatorname{dim}(G)=0$, so we can assume $n \geq 2$. Let $e=x y$ be an edge of $G$ ( $e$ is not a bridge), and let number of edges of $G$ parallel to $e$ (i.e. that have $x$ and $y$ as their ends). Now

$$
H_{2}(G)=H_{1}(G-e)+H_{2}(G \cdot e)
$$

(we recall that $G \cdot e$ denotes the contraction of edge $e$ in $G$ ).

If $\operatorname{dim}(G \cdot e) \geq 2$ and $G \cdot e$ is not the $K_{1}$-bond of two cycles, one of which has length 2 , then by induction on $n$,

$$
\begin{aligned}
H_{2}(G) & \geq H_{1}(G-e)+H_{1}(G \cdot e) \\
& \geq 1+\left(H_{1}(G)-1\right) \\
& =H_{1}(G)
\end{aligned}
$$

as $\operatorname{dim}(G-e)=\operatorname{dim}(G)-1 \geq 1$ since $G$ has no bridges.
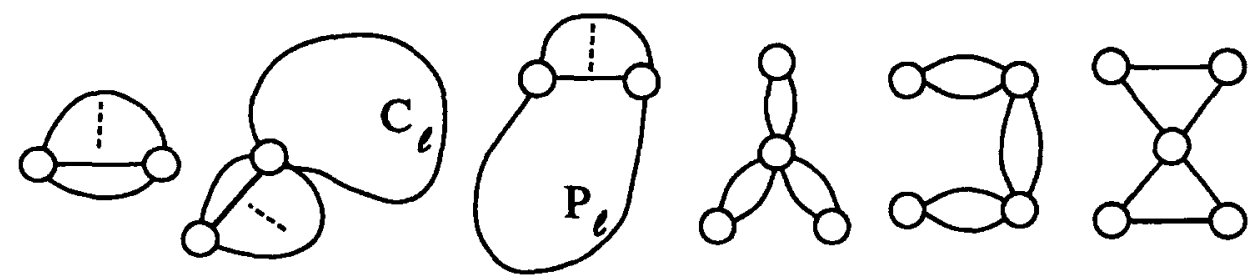

Figure I. 


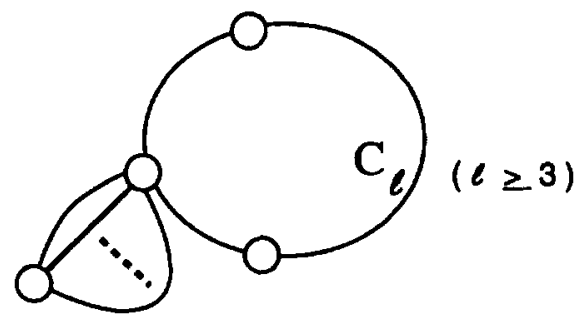

G

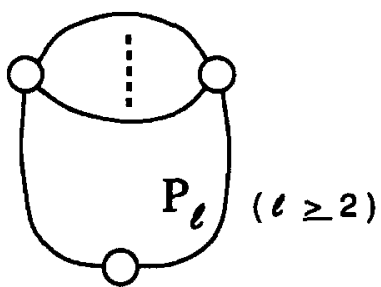

G

Figure 2.

In fact, equality can hold here if and only if $H_{2}(G \cdot e)=H_{1}(G \cdot e)$ and $H_{1}(G-e)=1$, i.e. $G-e$ (up to contraction of bridges) is a set of at least 2 parallel edges, so $G$ is of one of the two forms in Fig. 2, i.e. $G$ is the $K_{1}$-bond of a cycle of length at least 3 with one of its edges replaced by a set of at least 3 multiple edges, or a cycle of length at least 3 with one of its edges replaced by a set of at least 2 multiple edges (in the figure, $C_{l}$ and $P_{l}$ denote a cycel and path of length $l$ respectively).

The $H$-vectors are respectively $(1, \ell, \ell, \ldots, \ell, \ell-1)$ and $(1, \ell, \ldots, \ell, \ell)$. By assumption, $G$ is not of the forbidden form, so equality holds in this case if and only if $G$ if one of the graphs in Fig. 2.

The remaining cases are

(i) $\operatorname{dim}(G \cdot e)=2$ and $G \cdot e$ is of the forbidden form, or

(ii) $\operatorname{dim}(G \cdot e) \leq 1$.

We begin with Case (i). There are essentially 3 choices for which vertex is $\cdot e$ (see Fig. 3 ).

Subcase $I$. $G$ is of one of the forms in Fig. 4 (since $G$ is not of the forbidden form and $G$ has no bridges).

In (a)-(d), it is easy to verify that $H_{2}(G) \geq H_{1}(G)$, with equality if and only if $G$ is the $K_{1}$-bond of 3 cycles of length 2 . In case (e), $H_{2}(G) \geq H_{1}(G)$ (unless $\ell=2$ and $\mu(x, y)=1$, but this implies that $G$ is the $K_{1}$-bond of a 2-cycle and a 3-cycle, a contradiction), so here $H_{2}(G)>H_{1}(G)$.

Subcase II. $G$ is of one of the forms in Fig. 5 .

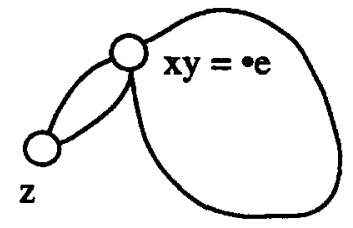

$\mathrm{G} \cdot \mathrm{e}$

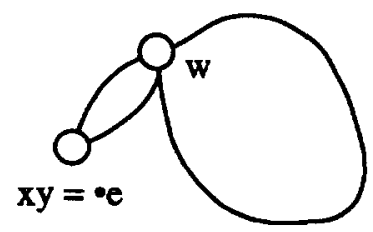

$\mathrm{G} \cdot \mathrm{e}$

II

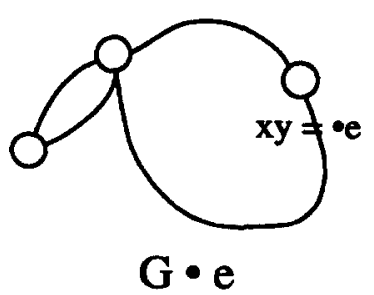

III

Figure 3. 


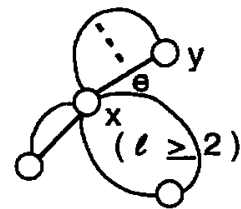

G

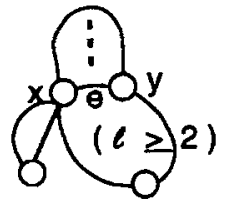

G

(b)

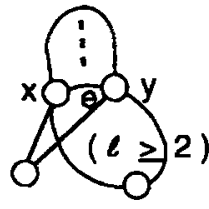

G

(c)

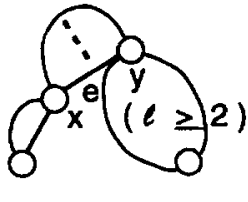

G

(d)

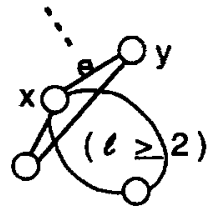

G

(e)

Figure 4.

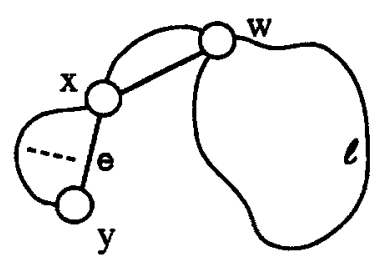

G

(a)

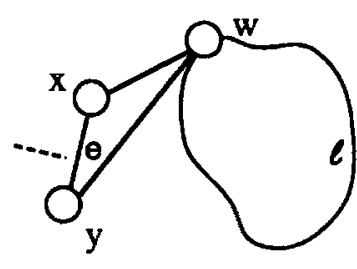

G

(b)

Figure 5.

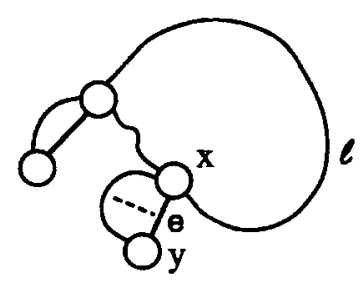

G

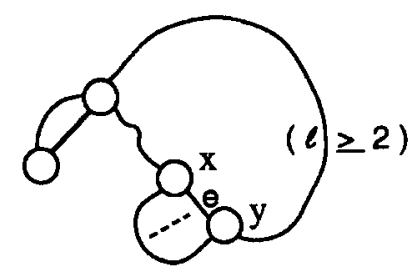

G

(b)

(a)

Figure 6.

In (a), $H_{2}(G) \geq H_{1}(G)$ (with equality if and only if $\ell=2$ and $G$ is the $K_{1}$-bond of three 2-cycles). In (b), $H_{2}(G) \geq H_{1}(G)$ (unless $\ell=2$ and $\mu(x, y)=1$, which implies $G$ is the $K_{1}$-bond of a 2-cycle and a 3 -cycle, a contradiction). Thus here $H_{2}(G) \geq H_{1}(G)$, with equality if and only if $G$ is the $K_{1}$-bond of two 3-cycles.

Subcase III. $\quad G$ is of one of the forms in Fig. 6 .

In (a) or (b), $H_{2}(G) \geq H_{1}(G)$.

We turn now to case (ii). First assume that $\operatorname{dim}(G \cdot e)=1$. As $G \cdot e$ is 2-edge connected, $G \cdot e$ is a cycle (with a loop at $\cdot e$ ), so $G$ is of one of the the forms in Fig. 7 .

In (a) the $H$-vector is $(1, \ell, \ell, \ldots, \ell, \ell-1)$, so $H_{2}(G)=H_{1}(G)$. In (b), we have seen that its $H$-vector is $(1, \ell, \ell, \ldots, \ell)$, so $H_{2}(G)=H_{1}(G)$. 


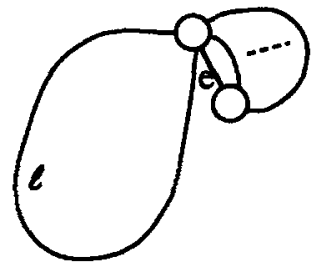

G

(a)

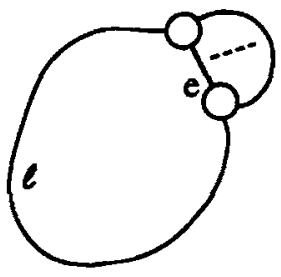

G

(b)

Figure 7.

If $\operatorname{dim}(G \cdot e)=0$, then $G$ is a group of at least 3 parallel edges, so $H_{2}(G)=H_{1}(G)=1$.

In fact, equality holds if and only if $G$ is of one of the forms in Fig. 1.

We turn our attention now to the second last term, $H_{d-1}$. The Stanley bounds imply an upper bound for $H_{d}$ in terms of $H_{d-1}$, but for shellable complexes, $H_{d}$ may be arbitrarily small. We show below that for cographic matroids, $H_{d}$ is bounded below by $H_{d-1} / d$.

Theorem 11 Let a connected graph $G$ have dimension $d$. Then

$$
H_{d-1}(G) \leq d H_{d}(G) \quad(*)
$$

and equality holds if and only if (except for loops) $G=k T$, where $T$ is a tree and $k=1$ or 2.

Proof: We proceed by induction on $m=|E(G)|$. If $m=n-1$, then $G$ is a tree, so $d=0$ and

$$
0=H_{d-1}(G) \leq d H_{d}(G)=0 \cdot 1,
$$

so equality holds.

Now assume $m>n-1$. Without loss, $G$ has no bridges (as if $e$ is a bridge, then $G$ and $G \cdot e$ have the same $H$-vectors but $G \cdot e$ has one fewer edge, so we are done by induction). Similarly, we can assume that $G$ is loopless. Let $e \in E(G)$. Then

$$
H_{d-1}(G)=H_{d-2}(G-e)+H_{d-1}(G \cdot e) .
$$

Now $\operatorname{dim}(G-e)=\operatorname{dim}(G)-1=d-1$ since $e$ is not a bridge or loop, so by induction on the number of edges,

$$
H_{d-2}(G-e)=(d-1) H_{d-1}(G-e) .
$$

From (2) and (3),

$$
H_{d-1}(G) \leq(d-1) H_{d-1}(G-e)+H_{d-1}(G \cdot e) .
$$

Let $e$ be parallel to $\mu$ other edges of $G$; it is not hard to see that

$$
\operatorname{dim}(G \cdot e)=d-\mu .
$$


Case 1. $\mu=0$.

Then $\operatorname{dim}(G \cdot e)=d$ by (5), so by induction on $m$,

$$
H_{d-1}(G \cdot e) \leq d H_{d}(G \cdot e) .
$$

Case 2. $\quad \mu \geq 2$.

Then $\operatorname{dim}(G \cdot e) \leq d-2$ by (5), so $H_{d-1}(G \cdot e)=H_{d}(G \cdot e)=0$, and thus

$$
H_{d-1}(G \cdot e) \leq d H_{d}(G \cdot e) .
$$

Thus in either case 1 or 2 , by (4), (6) and (7) we get

$$
\begin{aligned}
H_{d-1}(G) & \leq(d-1) H_{d-1}(G-e)+d H_{d}(G \cdot e) \\
& <d\left(H_{d-1}(G-e)+H_{d}(G \cdot e)\right) \\
& =d H_{d}(G)
\end{aligned}
$$

since as $G$ is loopless with no bridges, $d=m-(n-1)>0$ and $H_{d-1}(G-e)>0$ as $\operatorname{dim}(G-e)=d-1$.

Case 3. $\mu=1$. Since no edge of $G$ satisfies Case 1 or $2, G=2 F$ for some simple graph $F$. Now $F$ has no loops as $G$ has no loops, so

$$
d^{\prime} \equiv \operatorname{dim}(F)=|E(F)|-(|V(F)|-1)=\frac{m}{2}-(n-1) .
$$

Now

$$
\begin{aligned}
\operatorname{Rel}(G, p) & =\operatorname{Rel}\left(F, 1-(1-p)^{2}\right) \\
& =p^{n-1}(2-p)^{n-1} \sum_{i=0}^{d^{\prime}} H_{i}(F)(1-p)^{2 i}
\end{aligned}
$$

and so by considering the coefficient of $p^{m}=p^{d+n-1}=p^{2(n-1)+2 d^{\prime}}$ on both sides, we get

$$
H_{d}(G)=H_{d^{\prime}}(F)
$$

and

$$
H_{d-1}(G)=H_{d^{\prime}-1}(F)+(n-1) H_{d^{\prime}}(F) .
$$

Now by induction on $m$, we derive from (8) and (9) that

$$
\begin{aligned}
H_{d-1}(G) & \leq d^{\prime} H_{d^{\prime}}(F)+(n-1) H_{d^{\prime}}(F) \\
& \leq\left(d^{\prime}+(n-1)\right) H_{d^{\prime}}(F) \\
& =\frac{m}{2} H_{d}(G) \\
& \leq(m-(n-1)) H_{d}(G) \quad\left(\text { as } F \text { connected implies } \frac{m}{2} \geq(n-1)\right) \\
& =d H_{d}(G)
\end{aligned}
$$

and the inequality is proved. 
Now if $G$ is a connected and loopless graph for which equality holds in (*), then from above either $G$ is a tree, or $G=2 F$ for some simple graph $F$ and we must have equality in all the steps leading up to (10). That is,

$$
|E(F)|=2|E(F)|-(n-1)
$$

which implies

$$
|E(F)|=n-1
$$

so $G$ is a tree. It is not hard to see that in this case $G$ has dimension $n-1$ and $H$-vector $\left(1,\left(\begin{array}{c}n-1 \\ 1\end{array}\right),\left(\begin{array}{c}n-1 \\ 2\end{array}\right), \ldots,\left(\begin{array}{c}n-1 \\ n-2\end{array}\right), 1\right)$, and thus

$$
H_{d-1}(G)=(n-1)=(n-1) \cdot 1=d H_{d d}(G)
$$

and equality holds.

On the other hand, $H_{d-1}$ cannot be smaller than $H_{1}$. This inequality follows from Proposition 7.5.3 in [3]; the case of equality is examined next.

Theorem 12 For any graph $G$ of dimension $d \geq 2, H_{d-1} \geq H_{1}$, and equality holds if and only if $d=2$ or the underlying simple graph is a tree.

Proof: We proceed by induction on $m=|E(G)|$. If $m=n-1$, the result is trivial, so assume that $m>n-1$. We can assume that $G$ is loopless and bridgeless. Now for any edge $e$

$$
H_{d-1}(G)=H_{d-2}(G-e)+H_{d-1}(G \cdot e)
$$

so if $\operatorname{dim}(G \cdot e)=d$, that is, $e$ is not parallel to another edge, then

$$
\begin{aligned}
H_{d-1}(G) & \geq H_{d-2}(G-e)+H_{1}(G \cdot e) \\
& =H_{d-2}(G-e)+(n-2) \quad \text { (as } G \cdot e \text { has no bridges) } \\
& \geq n-1
\end{aligned}
$$

as $\operatorname{dim}(G-e)=d-1$.

The only remaining case is that every edge is parallel to at least one other edge. Now if $G$ is not twice some tree, then there is an edge $e$ of $G$ such that $G-e$ has no bridges. Also, $d=2$ is trivial, so we can assume $d \geq 3$, so by induction

$$
\begin{aligned}
H_{d-1}(G) & =H_{d-2}(G-e)+H_{d-1}(G \cdot e) \\
& \geq n-1+H_{d-1}(G \cdot e) \\
& \geq H_{1}(G) .
\end{aligned}
$$

If $G=2 T$ for some tree $T$, then $H_{d-1}=n-1=H_{1}$, so we are done.

For equality to hold, we need either $d=2$ or $d \geq 3$ and either (i) $e$ is not parallel to any other edges and $H_{d-2}(G-e)=1$, which implies by induction $H_{1}(G-e)=1$, so $G-e$ is a tree with one other edge parallel to some edge of $G$, and this implies $d=2$, a contradiction, or (ii) every edge is parallel to another edge, at least one edge $e$ is such that 
$G-e$ has no bridges and equality holds for $G-e$. By induction applied to $G-e$, either $d=3$ or the underlying simple graph of $G-e$ is a tree. In either case, $G$ must be a multiple of a tree (as the extra edge $e$ must be parallel to some other), and we are done.

Finally, as mentioned earlier, the $H$-vector of any graph whose underlying simple graph is a tree is symmetric, and hence if the dimension is at least two, then $H_{1}=H_{d-1}$.

\section{New bounds on the domination number}

The domination number (c.f. [4]) is the last non-zero term of the $H$-vector, namely $H_{d}$, where $d$ is the dimension of the graph; as noted earlier, $H_{d}$ is also a topological invariant of the matroid, the absolute value of its Euler characteristic. While some results are known for domination, we prove here some new bounds. These have implications to the Ball-Provan method for bounding network reliability.

Note that $H_{d}(G)=H_{d^{\prime}}\left(G^{\prime}\right)$, where $G^{\prime}$ is the underlying simple graph of $G$ and $d^{\prime}$ is its dimension.

Theorem 13 Let $G$ be a connected simple graph of dimension $d \geq 1$. Then

$$
H_{d}(G) \geq H_{1}(G)
$$

with equality if and only if $d=1$ or $G \equiv K_{3} \circ K_{3}$.

Proof: We proceed by induction on the size of $G$. We may assume that $G$ has no bridges. If $d=1$ then the result trivially holds, so we can assume that $d \geq 2$.

First assume that $G$ has blocks $G_{1}, \ldots, G_{k}$ with $k \geq 2$. Let the dimension of $G_{i}$ be $d_{i}$, which is at least 1 as $G$ has no bridges. Then $G$ simple implies that $H_{1}\left(G_{i}\right)=\left|V\left(G_{i}\right)\right|-1$ $\geq 2$,

$$
\begin{aligned}
H_{d}(G) & =\prod_{i=1}^{k} H_{d_{i}}\left(G_{i}\right) \geq \prod_{i=1}^{k} H_{1}\left(G_{i}\right) \\
\geq & \sum_{i=1}^{k} H_{1}\left(G_{i}\right)=H_{1}(G)
\end{aligned}
$$

where equality holds if and only if $k=2$ and $H_{1}(G)=H_{2}(G)=2$, i.e. $G \equiv K_{3} \circ K_{3}$.

Now assume $G$ is a block. If $e=x y$ is an edge of $G$ such that $G-e$ has no bridges, then

$$
\begin{aligned}
H_{d}(G) & =H_{d}(G \cdot e)+H_{d-1}(G-e) \\
& >H_{1}(G-e) \\
& =H_{1}(G)
\end{aligned}
$$

since $G \cdot e$ has dimension $d$ as $G$ is simple (and hence $H_{d}(G \cdot e)>0$ ). Otherwise, $G-e$ has a bridge, say $f=w z$. If $e$ and $f$ are non-adjacent, then $G \cdot e$ is simple, so

$$
\begin{aligned}
H_{d}(G) & =H_{d}(G \cdot e)+H_{d-1}(G-e) \\
& \geq H_{1}(G \cdot e)+H_{d-1}(G-e) \\
& >H_{1}(G)-1+H_{d-1}(G-e) \\
& >H_{1}(G)
\end{aligned}
$$


since $d-1 \geq 1$ implies that $G-e$ has a cycle of length at least 3 , and hence $H_{d-1}(G-e) \geq$ $H_{1}(G-e) \geq 2$. Finally, if $e$ and $f$ are adjacent, say $x=w$ (and as $G$ is simple, $y \neq z$ ), then as $G$ is a block, $x$ is of degree 2 in $G$. If $y z \notin E(G)$, then $G \cdot e$ is simple and we proceed as in the previous case. If $y z \in E(G)$, then $G-x$ has no bridges and the underlying simple graph of $G \cdot e$ is $G-x$, and so

$$
\begin{aligned}
H_{d}(G) & =H_{d}(G \cdot e)+H_{d-1}(G-e) \\
& =H_{d-1}(G-x)+H_{d-1}(G-x) \\
& =2 H_{d-1}(G-x) \\
& \geq 2\left(H_{1}(G)-1\right) \\
& >H_{1}(G)
\end{aligned}
$$

and for equality to hold, $G$ must have order 3 , i.e. $G=K_{3}$; this implies that $d=1$, a contradiction. Thus we are done.

(See also Proposition 7.5.3 in [3]).

Corollary 14 Let $G$ be a connected loopless graph of dimension $d \geq 1$. Then

$$
H_{d}(G)=1 \quad \text { if and only if } G \text { is a multiple of a tree }
$$

and

$$
H_{d}(G)=2 \quad \text { if and only if } G \text { is } \equiv \text { to a multiple of } K_{3} .
$$

Proof: Let $G^{\prime}$ be the underlying graph of $G$, and let $d^{\prime}=\operatorname{dim}\left(G^{\prime}\right)$. Without loss, $G^{\prime}$ has no bridges. If $G^{\prime}$ is a block that is not $K_{1}$ or $K_{3}$, then from Theorem $13, H_{d}(G)=$ $H_{d^{\prime}}\left(G^{\prime}\right) \geq H_{1}\left(G^{\prime}\right) \geq 3$. If $G^{\prime}$ has blocks $G_{i}$ of dimension $d_{i} \geq 1(i=1, \ldots, r \geq 2)$ with $G_{i}=K_{3}$ if and only if $i \leq \ell$, then

$$
\begin{aligned}
H_{d}(G) & =H_{d^{\prime}}\left(G^{\prime}\right)=2^{\ell} \prod_{i=\ell+1}^{r} H_{d_{l}}\left(G_{l}\right) \\
& \geq 2^{\ell} \prod_{i=\ell+1}^{r} H_{1}\left(G_{l}\right) \geq 2^{\ell} 3^{r-\ell}>2 .
\end{aligned}
$$

This completes the proof.

We are now ready to prove a new lower bound for the domination number of a graph in terms of its dimension.

Theorem 15 Let $G$ be a simple graph. Then

$$
H_{d}(G) \geq 2 d
$$

Proof: We can clearly assume $d \geq 2$ (as for $d=1, G$ is a cycle of length at least 3 ) and that $G$ has no bridges. We proceed by induction on $d$. If $G$ has blocks $G_{1}, \ldots, G_{k}(k \geq 2)$ 
with $G_{i}$ of dimension $d_{i} \geq 1$, then

$$
\begin{aligned}
H_{d}(G) & =\prod_{i=1}^{k} H_{d_{i}}\left(G_{i}\right) \geq \prod_{i=1}^{k} 2 d_{i} \\
& \geq \sum_{i=1}^{k} 2 d_{i}=2 d .
\end{aligned}
$$

Thus we can assume that $G$ is a block. If $e$ is an edge of $G$, then

$$
\begin{aligned}
H_{d}(G) & =H_{d}(G \cdot e)+H_{d-1}(G-e) \\
& \geq H_{d}(G \cdot e)+2(d-1) \geq 2 d
\end{aligned}
$$

unless $H_{d}(G \cdot e)=1$. By Corollary 14 , this implies that $G \cdot e$ is a multiple of a tree. In this case, as $G$ is a simple block, one can verify that $G$ must be $K_{2}+\overline{K_{n-2}}$, which has $H_{d}(G)=2^{n-2}$, and $d=n-2$. The result follows as $2^{n-2} \geq 2(n-2)$ for $n \geq 3$.

Corollary 16 If $G^{\prime}$ is the underlying simple graph of a connected graph $G$, then

$$
H_{d}(G) \geq 2 \operatorname{dim}\left(G^{\prime}\right) .
$$

Finally, we strengthen our results in terms of the girth of the graph.

Theorem 17 Suppose that $G$ is a simple connected graph of dimension $d$ and girth $g$, $g \geq 3$. Then

$$
H_{d}(G) \geq d(g-1) .
$$

Proof: We proceed by induction on the size of $G$. Clearly we can assume that $d \geq 1$, and since $H_{d}\left(C_{n}\right)=n-1$, we can assume in fact that $d \geq 2$. We can also as usual assume that $G$ has no bridges.

If $G$ is not a block, i.e. $G=G_{1} \circ G_{2}$, then without loss $\gamma\left(G_{1}\right) \geq \gamma\left(G_{2}\right)$. Let $G_{i}$ have dimension $d_{i} \geq 1$. Then $\gamma\left(G_{1}\right) \geq g$ and we have

$$
\begin{aligned}
H_{d}(G) & =H_{d_{1}}\left(G_{1}\right) H_{d_{2}}\left(G_{2}\right) \\
& \geq d_{1}(g-1) H_{d_{2}}\left(G_{2}\right) \\
& \geq d_{1}(g-1)\left(2 d_{2}\right) \\
& \geq\left(d_{1}+d_{2}\right)(g-1) \\
& =d(g-1)
\end{aligned}
$$

where we use Theorem 15 to go from line 2 to line 3.

Thus we can assume that $G$ is a block. If $G \cdot e$ is simple for some edge $e$ of $G$, i.e. $e$ is not in a triangle of $G$, then as $G-e$ and $G \cdot e$ are of girth at least $g$ and $g-1$ respectively,

$$
\begin{aligned}
H_{d}(G) & =H_{d-1}(G-e)+H_{d}(G \cdot e) \\
& \geq(d-1)(g-1)+d(g-2) \\
& \geq d(g-1)
\end{aligned}
$$

as $d \geq 2$ and $g \geq 3$. 

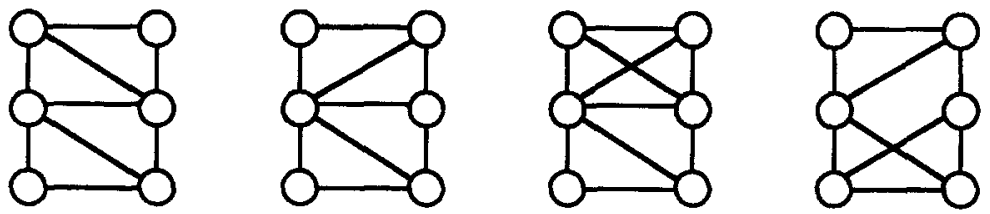

Figure 8 .

The only remaining case is that $G$ is a block and every edge of $G$ belongs to a triangle. Thus $g=3$, and if $e$ is any edge of $G$,

$$
\begin{aligned}
H_{d}(G) & =H_{d-1}(G-e)+H_{d}(G \cdot e) \\
& \geq(d-1) 2+2 \\
& =d(3-1)
\end{aligned}
$$

by Theorem 15 unless the underlying simple graph of $G \cdot e$ is $K_{3} \circ K_{3}$. As $G$ is a block with every edge in a triangle, one can verify that the only possibilities for $G$ are shown in Fig. 8. In each case the inequality holds, so we are done.

\section{Non-Stanley bounds for graphs of low dimension}

According to the Stanley bounds, the best upper bounds for $H_{d}$ of a shellable complex of fixed dimension $d$ is

$$
H_{d} \leq\left(\begin{array}{c}
H_{1}-1+d \\
d
\end{array}\right) \sim \frac{H_{1}^{d}}{d !} .
$$

with equality obtained by taking a uniform matroid. However, for cographic matroids, the results are different.

Theorem 18 If $G$ is a connected graph of dimension $d \leq 2$, then

$$
H_{2}(G) \leq \frac{1}{3}\left(H_{1}^{2}(G)+H_{1}(G)+1\right)
$$

and equality holds if and only if $d=0$, or $d=2$ and $G$ is a $\theta$-graph with 3 equal length paths.

Proof: If $d<2$ then $H_{2}(G)=0$, so the result is trivial. Assume now $d=2$. We can also assume $G$ has no bridges, as contracting a bridge leaves the $H$-vector unchanged. Thus every vertex has degree at least 2 . Let $G$ have order $n$ and size $m$ (so $d=m-n+1)$ with $x$ vertices of degree $\geq 3$. Then

$$
\begin{aligned}
2 m & =\sum_{v \in V(G)} \operatorname{deg}(v) \\
2(d+n-1) & \geq 3 x+2(n-x) \\
2 n+2 & \geq 2 n+x \\
x & \leq 2 .
\end{aligned}
$$




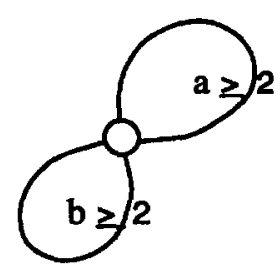

G

(a)

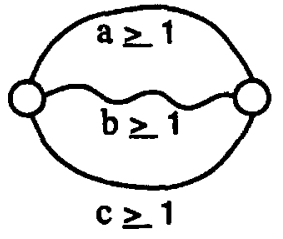

G

(b)

Figure 9.

Thus either $x=1$ and the only vertex of degree greater than two has degree 4 , or $x=2$ and the two vertices of degree greater than two have degree 3. It follows that $G$ is of one of the two forms shown in Fig. 9.

In (a), $G=C_{a} \circ C_{b}(a, b>1)$ has $H$-vector $(1, a+b-2, a b-a-b+1)$. Now for fixed $a+b$ (and hence fixed $H_{1}(G)=a+b-2$ ), $a b-a-b+1$ is maximized when $a b$ is maximized, i.e. when $a=b=\frac{H_{1}+2}{2}$. Therefore,

$$
\begin{aligned}
H_{2}(G) & \leq\left(\frac{H_{1}(G)+2}{2}\right)^{2}-2\left(\frac{H_{1}(G)+2}{2}\right)+1 \\
& \leq \frac{H_{1}^{2}(G)}{4}<\frac{1}{3}\left(H_{1}^{2}(G)+H_{1}(G)+1\right) .
\end{aligned}
$$

In (b), $G$ (a $\theta$-graph with paths of lengths $a, b, c$, each os size at least 1 ) has $H$-vector $(1, a+b+c-2, a b+a c+b c-a-b-c+1)$. Now for fixed $a+b+c$ (and hence for fixed $\left.H_{1}(G)=a+b+c-2\right), a b+a c+b c-a-b-c+1$ is maximized when $a b+a c+b c$ is, i.e. when $a=b=c=\frac{H_{1}(G)+2}{3}$. Thus

$$
\begin{aligned}
H_{2}(G) & \left.\leq \frac{1}{3}\left(H_{1}(G)+2\right)^{2}-\left(H_{1}(G)+2\right)+1\right) \\
& \leq \frac{1}{3}\left(H_{1}^{2}(G)+H_{1}(G)+1\right) .
\end{aligned}
$$

In fact, equality holds only in (b), and then only if $a=b=c$, i.e. $G$ is a $\theta$-graph with 3 equal length paths.

Such a bound lifts to produce non-Stanley bounds for all fixed dimensions. For example,

Corollary 19 If $G$ is a connected graph of dimension $d \leq 3$, then

$$
H_{3}(G) \leq \frac{1}{9}\left(H_{1}^{3}(G)+3 H_{1}^{2}(G)+5 H_{1}(G)+3\right)
$$

with equality if and only if $d=0$.

Proof: We proceed by induction on $n$, the number of vertices of $G$. Clearly, we can assume $d=3$ and that $G$ has no bridges. If $n=1$ we are done, so we can assume that 


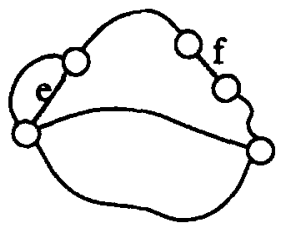

G

(a)

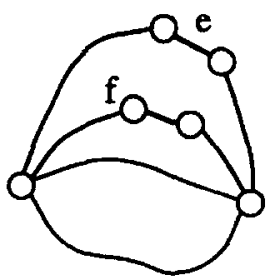

G

(b)

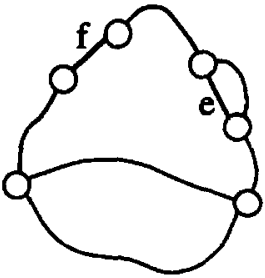

G

(c)

Figure 10.

$n \geq 2$. Now if $e \in E(G)$, then

$$
\begin{aligned}
H_{3}(G)= & H_{2}(G-e)+H_{3}(G \cdot e) \\
\leq & \frac{1}{3}\left(H_{1}^{2}(G-e)+H_{1}(G-e)+1\right) \\
& +\frac{1}{9}\left(H_{1}^{3}(G \cdot e)+3 H_{1}^{2}(G \cdot e)+5 H_{1}(G \cdot e)+3\right) .
\end{aligned}
$$

Now $H_{1}(G-e) \leq H_{1}(G)=n-1$ and $H_{1}(G \cdot e)=H_{1}(G)-1=n-2$, so

$$
\begin{aligned}
H_{3}(G) \leq & \frac{1}{3}\left((n-1)^{2}+(n-1)+1\right) \\
& +\frac{1}{9}\left((n-2)^{3}+3(n-2)^{2}+5(n-2)+3\right) \\
\leq & \frac{1}{9}\left((n-1)^{3}+3(n-1)^{2}+5(n-1)+3\right) \\
\leq & \frac{1}{9}\left(H_{1}^{3}(G)+3 H_{1}^{2}(G)+5 H_{1}(G)+3\right) .
\end{aligned}
$$

In fact, if equality were to hold, then we must have equality holding everywhere above, so in particular, $\left.H_{2}(G-e)=\frac{1}{3}\left(H_{1}(G-e)+2\right)^{2}+\left(H_{1}(G-e)+2\right)+1\right)$. From the previous theorem, this means $G-e$ is a $\theta$-graph with 3 equal length paths of length $a \geq 1$. Also, $\operatorname{dim}(G \cdot e)=3$, so (as $e$ was arbitrary), $G$ must be a simple graph. Now $a=1$ implies that $G$ is a bundle of parallel edges, so $H_{2}(G)=H_{1}(G)=1$ and $\frac{1}{9}\left(H_{1}^{3}(G)+3 H_{1}^{2}(G)+\right.$ $\left.5 H_{1}(G)+3\right)=\frac{4}{3}$, so strict inequality holds. Thus $a \geq 2$ and $G$ is of one of the forms in Fig. 10.

If we choose $f$ instead of $e$ in (a) or (b), $G-e$ (after contracting bridges) is not a $\theta$-graph. If we choose $f$ instead of $e$ in (c), then $G-f$ is a $\theta$-graph with path lengths $1, a \geq 2, a \geq 2$, so we can see in any event, we can choose an edge $e$ so that $H_{2}(G-e)<$ $\frac{1}{3}\left(H_{1}^{2}(G-e)+H_{1}(G-e)+1\right)$. Thus (provided $d \geq 1$ ) equality never holds in the corollary.

We expect that the results for lower dimensions can be drawn up to higher dimensions to improve bounds between terms that are close to one another in the $H$-vector of a graph. 


\section{Acknowledgments}

The authors wish to thank the referees for their detailed suggestions.

\section{References}

1. M.O. Ball and J.S. Provan, "Bounds on the reliability polynomial for shellable independence systems," SIAM J. Alg. Disc. Meth. 3 (1982), 166-181.

2. L.J. Billera and C.W. Lee, "A proof of the sufficiency of McMullen's conditions for $f$-vectors of simplicial convex polytopes," J. Comb. Th. A 31 (1981), 237-255.

3. A. Björner, "Homology and Shellability," in Matroid Applications, N. White (Ed.), Cambridge University Press, Cambridge, 1992.

4. F.T. Boesch, A. Satyanarayana, and C.L. Suffel, "Least reliable networks and the reliability domination," IEEE Trans. Comm. 38 (1990), 2004-2009.

5. J.I. Brown and C.J. Colbourn, "A set system polynomial with colouring and reliability applications," SIAM J. Disc. Math. 1 (1988), 151-157.

6. J.I. Brown and C.J. Colbourn, "On the log concavity of reliability and matroidal sequences," Adv. Appl. Math. 15 (1994), 114-127.

7. J.I. Brown and C.J. Colbourn, "Roots of the reliability polynomial," SIAM J. Disc. Math. 5 (1992), 571-585.

8. J.I. Brown, C.J. Colbourn, and J.S. Devitt, "Network transformations and bounding network reliability," Networks 23 (1993), 1-17.

9. C.J. Colbourn, The Combinatorics of Network Reliability, Oxford Univ. Press, New York, 1987.

10. C.J. Colbourn and W.R. Pulleyblank, "Matroid Steiner Problems, the Tutte polynomial and network reliability," J. Comb. Th. B 47 (1989), 20-31.

11. T. Hibi, "Face number inequalities for matroid complexes and Cohen-Macaulay types of Stanley-Reisner rings of distributive lattices," Pacific J. Math. 154 (1992), 253-264.

12. F.S. Macaulay, "Some properties of enumeration in the theory of modular systems," J. Lond. Math. Soc. 26 (1927), 531-555.

13. R.P. Stanley, "Cohen-Macaulay complexes," in Higher Combinatorics, M. Aigner (Ed.), Reidel, 51-64, 1977.

14. R.P. Stanley, Combinatorics and Commutative Algebra, Birkhäuser, Boston, 1983.

15. W.T. Tutte, “A ring in graph theory," Proc. Cambridge Phil. Soc. 43 (1947), $26-40$.

16. D.J.A. Welsh, Matroid Theory, Academic Press, London, 1976. 